
profesionales

\title{
Contribuciones del enfoque poblacional a la seguridad social
}

Contributions of the population-based approach to social security

Cecilia Molina

Licenciada en Trabajadora Social

(Universidad Nacional de Córdoba)

Máster en Salud pública y gestión sanitaria

(Escuela Andaluza de Salud Pública y

Universidad de Granada, España)

Doctora en Ciencias Sociales

(Universidad Nacional de Cuyo)

Docente en Facultad de Ciencias Políticas y

Sociales

(Universidad Nacional de Cuyo)

Correo: cecilia.molina@osep.mendoza.gov.ar 


\title{
Resumen
}

Este escrito sintetiza una estrategia iniciada hace cuatro años para contribuir a adoptar la mirada poblacional en la Obra Social de Empleados Públicos (OSEP), en Mendoza, Argentina. Puntualiza los desafíos que conlleva gestionar la salud de cerca de 400.000 personas que trabajan en la administración pública y sus familias desde una perspectiva que cuestiona modelos de financiamiento y de atención fuertemente arraigados en la seguridad social, cuya base son los intereses de la oferta prestacional. Se comparten el contexto de la experiencia, las nociones teóricas que orientan las líneas de trabajo diseñadas para contemplar los escenarios donde transcurre la salud y la enfermedad de las personas afiliadas a la obra social y la metodología empleada, poniendo de relieve la tarea de les promotores territoriales de salud. Se presentan resultados y se reflexiona sobre la adopción de la estrategia en el desempeño global de la organización. A cuatro años de iniciada la tarea, podemos expresar en indicadores los modos desiguales como se tramitan los procesos de salud/enfermedad/atención en los territorios en los que viven y/o trabajan las familias aseguradas y dar cuenta de dispositivos diseñados para avanzar en respuestas receptivas a sus especificidades. Concluimos en que profundizar en la ruta propuesta supone aprendizajes organizacionales de largo alcance.

\section{Palabras clave}

Gestión poblacional, Territorio, Seguridad social.

\begin{abstract}
This text summarizes a strategy initiated four years ago in order to contribute to adopt the population's view in the Social Work of Public Employees (OSEP) in Mendoza, Argentina. It identifies the challenges that lie ahead the health management of around 400.000 public employees and their families from a perspective that questions financial and attendance modelsfirmly attached to social security, which basis are the interests of medical providers. The context of the experience is shared, also the theoretical notions which orientate the major lines of work design to contemplate the scenarios where health and diseases of the affiliates occur and the methodology used, highlighting the job of territorial health promoters. Results are presented and thoughts about the global organizational performance of the adoption of this strategy are included. After four years of the beginning of this work, we can express through indicators the unequal modes in how health/disease/attention processes are developed in the territories where affiliated families live and/or work and expose designed devices to the proceed in receptive answers linked to their specificities. We conclude that getting deeper in the proposed route requires long-range organizational learnings.
\end{abstract}

\section{Keywords}

Population management, Territory, Social security,. 


\section{Introducción}

La adecuación de las respuestas sanitarias a las condiciones de vida, a las necesidades y demandas de las personas que están estrechamente ligadas a las particularidades de los territorios que habitan es un asunto apenas problematizado en las organizaciones de seguridad social en Argentina.

En general, las obras sociales, que son las responsables de garantizar la atención de la salud de cerca de la mitad de les habitantes del país ${ }^{1}$, estructuran sus prioridades financieras y prestacionales sin atender a las especificidades socioeconómicas, demográficas y epidemiológicas de las poblaciones a su cargo. En concordancia con el modelo biomédico dominante, conciben el proceso de salud/enfermedad/atención como una cuestión individual, asistencialista y ahistórica (Menéndez, 1990) y desconocen que los modos de vivir, enfermar y morir, si bien son atravesados por dinámicas globales, se expresan de manera desigual en los territorios (Molina Jaramillo, 2018; Borde y Torres Tovar, 2017).

A contrapelo de los enfoques centrados en los servicios curativos y en los programas focalizados, característicos del neoliberalismo sanitario, en los últimos años gana aceptación la idea de que la salud está socialmente determinada (López Arellano, Escudero y Dary Carmona, 2008). Se ha vuelto a poner la mirada en perspectivas horizontales, que asumen que se produce más salud organizando respuestas por niveles de complejidad e intentan ser receptivas a las realidades territoriales (Tobar, 2008).

Sustenta este planteo la comprensión de los territorios como escenarios de relaciones y conflictos que impactan en las condiciones de vida de las personas y en sus posibilidades de acceso a los servicios de salud ${ }^{2}$ y la evidencia de que las dinámicas territoriales condicionan la disponibilidad y

1 Según el Análisis de situación de salud 2018 del Ministerio de Salud de la Nación, 64\% de la población cuenta con cobertura de obra social o privada, siendo la obra social la cobertura mayoritaria (46\% del total), mientras que $36 \%$ de la población se atiende exclusivamente en servicios públicos de salud.

2 Mientras que para la salud pública tradicional el territorio se asimila a demarcaciones políticas administrativas o a las condiciones del ambiente físico y los elementos de la naturaleza que inciden en la salud de las poblaciones, las corrientes que incorporan nociones de la geografía social crítica asumen que el territorio se construye a partir de relaciones históricas, sociales, culturales, económicas, políticas y ambientales, configurando el escenario donde se expresan contradicciones y donde pulsa la vida, la salud, la enfermedad y la muerte" (Borde y Torres Tovar, 2017:272-273). 
articulación entre les actores con injerencia en la prestación de los servicios (Chiara, 2016).

El enfoque poblacional de la salud incorpora la perspectiva territorial y procura integrar respuestas haciendo que la oferta de bienes y servicios de salud se aproxime a las necesidades de poblaciones identificadas con claridad. Para ello, prioriza el conocimiento y el vínculo activo con esas poblaciones (con independencia de que demanden respuestas a los servicios asistenciales), promueve la distribución precisa de responsabilidades de los equipos de salud y la gestión y evaluación con base en resultados (Tobar, 2010). Estas modalidades son afines a discursos vigentes en la seguridad social pero no siempre logran incorporarse a las decisiones y acciones cotidianas de estas organizaciones.

Este escrito sintetiza la estrategia diseñada y ejecutada durante cuatro años por un equipo de trabajo interdisciplinario guiado por el objetivo de desarrollar el enfoque territorial y poblacional en la Obra Social de Empleados Públicos (OSEP) de Mendoza, Argentina, y los avances y limitaciones identificados en el proceso para adoptar, en diferentes niveles de la organización, las lógicas asistenciales y de gestión que abonan esta perspectiva. El escrito se organiza en cuatro apartados. Primero, se caracteriza el contexto en el que se inscribe la experiencia. Luego se explicitan los aspectos metodológicos. En tercer lugar, se presentan resultados y se cierra con reflexiones.

\section{El contexto de la experiencia}

Las obras sociales en Argentina son parte de un sistema de salud fragmentado en su financiamiento, segmentado en la provisión de los servicios y profundamente inequitativo en el acceso a la atención médica. A diferencia de los sistemas universales europeos y de algunos sistemas de seguridad social de países latinoamericanos que en la última década avanzaron en reformas tendientes a integrar flujos y funciones, ${ }^{3}$ la seguridad social, en el

3 Costa Rica, Brasil, Colombia, Uruguay y Perú son para Cetrángolo y Goldschmit (2018) países que, teniendo fuentes de financiamiento y de provisión mixtas como en Argentina, están más adelantados en la integración de aseguramientos públicos y otros sistemas de protección social. 
país, "mantiene un patrón divergente en el acceso y la cobertura de salud" (Cetrángolo y Goldschmit, 2018:3). El subsector de obras sociales, a su vez, alberga significativas diferencias, que se explican en parte por los diferentes recursos que disponen, según el ingreso promedio de sus contribuyentes.

La forma de organización político-administrativa del país agrava las inequidades de cobertura sanitaria, ya que las zonas con más necesidad de atención médica cuentan con menor financiamiento público y de la seguridad social (De Ponti, Canale y Monteferrario, 2016).

En este escenario heterogéneo, las obras sociales provinciales ${ }^{4}$ (entre ellas la OSEP) deben garantizar el acceso igualitario a la salud a les trabajadores estatales provinciales y sus familias, quienes representan el 16\% de la población de la Argentina. Se orientan por el principio de solidaridad, según el cual cada afiliado aporta en relación con su ingreso y recibe según las prestaciones que necesite, pero no se asignan los mismos recursos a la salud de las personas a su cargo. ${ }^{5} \mathrm{Si}$ bien, en general, son autárquicas y manejan sus presupuestos con independencia de los ministerios de salud de sus respectivas provincias, suelen estar sujetas a las políticas de empleo y de ingresos y a las restricciones fiscales de los gobiernos subnacionales (Cetrángolo y Goldschmit, 2018).

La OSEP cubre a $21 \%$ de la población de Mendoza ${ }^{6}$. Por volumen de personas aseguradas y por peso en el mercado local de servicios de atención médica, tiene un rol protagónico en la salud en la provincia. Debe garantizar prestaciones a casi 400.000 personas que, si bien comparten el atributo de tener relación directa (titulares) o indirecta (sus familiares) con el Estado provincial y los 18 estados municipales, son profundamente heterogéneas

4 Las 24 obras sociales provinciales se agrupan en el Consejo de Obras y Servicios Sociales Provinciales de la República Argentina (COSSPRA), que tiene 7.200.000 asegurados. Más información se puede consultar en: _

5 Los salarios de los empleados públicos y las contribuciones patronales difieren entre los estados provinciales. Según Del Ponti y otros (2016), los aportes oscilan entre 8 \% y 14,5 \% del sueldo y en el financiamiento de la obra social por asegurado hay una distancia de 2,5 veces del mayor respecto al menor. Estas diferencias en el financiamiento no tienen relación directa con la accesibilidad, medida por tasa de uso en consultas y costos de las mismas.

6 La proporción de asegurados respecto de la población total presenta variaciones que van entre $13 \%$ y $39 \%$ según el departamento de residencia y $24 \%$ de los titulares tienen otra obra social además de OSEP. 
en cuanto al tipo de trabajo que realizan ${ }^{7}$, situación socioeconómica, capital social, necesidades, expectativas y demandas en salud.

En cuanto al perfil demográfico, OSEP tiene una población con marcada tendencia al envejecimiento, al extender sus protecciones a los jubilados de las administraciones provincial y municipal ${ }^{8}$. Es significativa la proporción de personas afiliadas mayores que viven solas, lo cual tensiona los dispositivos de cuidado tradicionales diseñados para ese grupo de edad.

La OSEP tiene presencia en los 18 departamentos en los que se divide la provincia y en algunos de los principales distritos que los componen. Su estructura de provisión es mixta: mediante convenios con organizaciones privadas y públicas y a través de servicios de su propiedad ${ }^{9}$, con variaciones según regiones. Esto es así porque, mientras más lejos de los centros urbanos viven las personas afiliadas, más dependen de la oferta asistencial pública $^{10}$, mientras que la oferta privada se concentra en los principales núcleos urbanos, donde se localizan los servicios de diagnóstico y tratamiento de mayor rentabilidad. Este panorama complejiza la gestión de la obra social y desafía la equidad. Como bien advierte Chiara:

La política institucional, en su implementación, se ve afectada por las particularidades de los mercados, por los mecanismos de compraventa de servicios que difieren según el tipo de prestación y por la dinámica que los actores imprimen en cada territorio, en función de sus particulares intereses, perspectivas y capacidades de reclamo (2016:19).

7 Pensemos qué tiene en común en términos de condiciones de vida que determinan la salud una jueza de la Suprema Corte con un trabajador municipal afectado a la recolección de residuos, un docente rural y una agente penitenciaria que cumple turnos rotativos.

8 En este aspecto, la mayor parte de las obras sociales provinciales difieren de las obras sociales nacionales y sindicales, cuyos asegurados una vez que se jubilan pasan a PAMI-ISSJP (Instituto Nacional de Seguridad Social para Jubilados y Pensionados).

9 La provisión mixta es una particularidad de OSEP, respecto de otras OSP y nacionales. Además de 2 hospitales que suman 240 camas (de adultos y pediátricas) tiene un Centro Odontológico, 18 sedes departamentales y más de 20 programas asistenciales y preventivos a cargo de personal propio.

10 En su condición de prestador directo, el sector público local aglutina en 24 hospitales el mayor porcentaje de camas de la provincia y cerca de 300 centros de salud; concentra la oferta de emergencia y los centros de internación de salud mental, geriatría y pediatría de alta complejidad. En zonas alejadas de la capital, monopoliza estos servicios. 


\section{Aspecto operativo de la experiencia}

El equipo de trabajo

La estrategia de abordaje poblacional es llevada adelante por profesionales de la sociología, la politología, trabajadores sociales, de la medicina de familia, de la comunicación social y por promotores territoriales de salud ${ }^{11}$. Les promotores despliegan su tarea en distritos y barrios de distintos puntos de la provincia y en reparticiones públicas priorizadas en base a análisis de información disponible en los sistemas de la organización ${ }^{12}$, criterios de vulnerabilidad institucionalmente concertados y acuerdos con las autoridades regionales de OSEP.

Si bien se privilegia el trabajo barrial, se opta por contactar en sus lugares de trabajo a las personas afiliadas, que tienen regímenes horarios que dificul$\tan$ localizarlas en sus viviendas ${ }^{13}$.

La tarea central de les promotores territoriales es actuar como puente entre las familias aseguradas y la organización. Coordinan su tarea con equipos de salud departamentales, con unidades de atención familiar (UAF) y con referentes de las líneas de cuidado priorizadas por la organización: Nacer, Crecer, Ser Mujer y Ser Mayor ${ }^{14}$.

Otra parte del equipo analiza información secundaria, acompaña el trabajo en terreno y la producción de informes poblacionales y se encarga de las coordinaciones que demanda la tarea en el campo.

11 Una parte de estos promotores son egresados de una tecnicatura universitaria mientras que otros son trabajadores que cumplían distintas tareas en OSEP y se sumaron al proyecto. Para formarlos nos han resultado valiosas las producciones de la Universidad Nacional de General Sarmiento.

12 El Sistema de Afiliaciones permite conocer edades, domicilios, repartición en la que trabaja en titular, afiliados indirectos a cargo e ingresos mientras que el SISAO es un sistema de registro de transacciones asistenciales en línea que posibilita identificar consultas, prácticas, prescripción de recetas y diagnósticos. 13 Por ejemplo, los trabajadores de Hidráulica, de Vialidad Provincial o los docentes y celadores de escuelas albergue que funcionan en el Secano.

14 Atención materno-infantil; atención de niños y adolescentes, detección precoz del cáncer femenino y prestaciones sociosanitarias para la tercera edad. 
Técnicas para la obtención y gestión de la información a partir de los problemas que se identifican

En los relevamientos en terreno se emplean cuestionarios (a las unidades familiares), entrevistas (a informantes y a referentes de organizaciones con base territorial) e información secundaria previamente procesada. Con base en los registros informatizados que proveen el Padrón de personas afiliadas y el Sistema de Salud de OSEP (SISAO), se mapean las desigualdades de ingresos de las personas titulares, se caracteriza demográficamente a las personas a contactar, se identifica la no utilización de servicios por grandes grupos de edad y se nominaliza a las familias con las que se establecerá relación. Esta información se plasma en hojas de ruta, que ordenan los procesos de inserción y de relevamiento por zona.

Los cuestionarios tienen un bloque en el que se indagan determinantes sociales de la salud familiar (características de las viviendas, problemas ambientales a las que están expuestas, acceso a los sistemas de transporte público, carga laboral de todos sus integrantes) y bloques con preguntas específicas para identificar problemas sociosanitarios, relaciones con los servicios de salud de proximidad y necesidades de cuidado de niñes, mujeres, adultes mayores y personas con discapacidad. El formulario de los cuestionarios tiene soporte en un aplicativo que posibilita la carga on-line a través de tablets.

A medida que realizan los relevamientos, les promotores clasifican a las familias según niveles de vulnerabilidad ${ }^{15}$, disparan alertas y despliegan intervenciones, cuando es necesario, con apoyo de mesas interdisciplinarias locales ${ }^{16}$ que discuten e intervienen en la resolución de situaciones críticas.

15 Esta clasificación está en sintonía con criterios de vulnerabilidad consensuados para los distintos grupos de edad desde distintos sectores de OSEP. En la línea que plantea Araujo González (2015:.92) entendemos la vulnerabilidad como el resultado de una combinación de eventos, procesos o rasgos que entrañan adversidades potenciales para el ejercicio de derechos ciudadanos y para el logro de proyectos colectivos, familiares y personales. Para esta óptica, "la vulnerabilidad individual y colectiva depende de características demográficas individuales, como edad, género o nivel educativo; de la composición del hogar, ciclo de vida, nivel socioeconómico, estatus ocupacional y situación de pobreza familiar, y de indicadores macrosociales, como el nivel de desarrollo y los contextos económicos y sociales, entre otros".

16 Según la complejidad de las unidades de atención familiar de los barrios, en el Gran Mendoza, o de las sedes territoriales en el interior están integradas por médicos de familia, pediatras, psicólogos y trabajadores sociales. La coordinación general de estos espacios está a cargo de una trabajadora social. 
Los problemas detectados que no tienen relación directa con la atención médica (por ejemplo, adolescentes excluidos del sistema educativo, déficits en el sistema público de recolección de residuos o transporte o sobrepoblación de perros callejeros en un barrio), dan lugar a coordinaciones con otras organizaciones de política social.

Técnicas para la sistematización y el análisis de datos

Los resultados de los cuestionarios a las familias se vuelcan en una base de información que alimenta el tablero de mando de la OSEP y en Análisis de Situación de Salud locales (ASIS). Esta herramienta permite identificar y priorizar problemas de salud presentes en los territorios relevados y desarrollar acciones que contribuyan a su solución, incorporando los recursos disponibles para dar respuesta a las necesidades de los grupos (Pría Barros, 2006). Estos informes incluyen mapeos de activos para la salud, en los que se consignan los recursos grupales, físicos, culturales y organizacionales que constituyen un capital social y pueden ser activados para sostener la salud y el bienestar de les afiliades y las familias que viven en los territorios trabajados (García et al, 2019).

Las situaciones de vulnerabilidad familiar que se identifican se registran en una herramienta informática denominada Trello ${ }^{17}$, que permite ordenar las intervenciones de los equipos locales de salud, de otras áreas de OSEP 18 o de otras organizaciones de política social.

17 Trello es un software de administración de proyectos que permite organizar tareas, adjuntar archivos y etiquetar alarmas. Puede ser consultado simultáneamente por equipos de trabajo físicamente distantes unos de otros.

18 Por los problemas identificados son frecuentes los contactos con Cuidados Domiciliarios, Planes Especiales, Trabajo Social, la Red de Rehabilitación, el Programa de Crónicos y Ser Mayor y el Departamento de Insumos y suministros. 
Formas de transferir la información a los diferentes niveles decisorios de la organización

La información que se produce tiene diferentes personas destinatarias. Los ASIS de barrios y distritos se vuelcan en la biblioteca virtual de la web de OSEP para que sean accesibles a los equipos locales de salud y a les directives. Los diagnósticos de situación por segmentos poblacionales (niñes de 1 a 14 años, por ejemplo) se transfieren a les responsables de las líneas de cuidado y a sus referentes territoriales. Cuando es posible, se procura presentar y discutir las producciones escritas en espacios colectivos. De esta manera se facilita la reflexión y la apropiación de los análisis de salud entre les potenciales utilizadores.

La información agregada que permite dar cuenta de perfiles de población a tener en cuenta al momento de planificar o convenir servicios con terceros se incorpora al tablero de comando de la organización.

Si bien el trabajo territorial que se realiza desde el área no tiene como propósito central gestionar reclamos, los malestares, las desinformaciones y las quejas que emergen en el transcurso de las entrevistas que realizamos, si no pueden ser resueltas por les promotores, se canalizan y transmiten a las áreas pertinentes.

\section{Principales resultados}

1. La incorporación de les promotores de salud ha permitido construir vínculos más estrechos con les asegurades y los contextos en los que transcurren sus vidas.

Si bien estamos muy lejos de llegar mediante esta figura a todes les afiliades, entre mediados de 2016 y diciembre $2019^{19}$, relevamos 8.935 familias (29.000 personas) con residencia en 192 distritos y 207 barrios de 14 de los 18 departamentos de la provincia. El impacto del trabajo de les promotores presenta variaciones según zonas. Oscila entre el 18\% del padrón de titulares 
en departamentos con características de ruralidad, como Lavalle y La Paz, a 3\% en los departamentos densamente poblados de Gran Mendoza.

Cerca del 20\% de las familias contactadas presentaron situaciones evaluadas como de vulnerabilidad alta y media. Fueron abordadas con los equipos asistenciales de apoyo en mesas interdisciplinarias. Situaciones recurrentes que han disparado intervenciones son: niños sin controles de salud previstos para su edad, adultes mayores sin apoyo social para resolver cuidados relacionados con su vida cotidiana y familias de trabajadores de servicios públicos con muy bajos niveles de ingreso que desconocen cómo acceder a prestaciones básicas para todes o algunes de sus integrantes.

La opción por considerar unidades de análisis y de intervención a las familias (en vez de los individuos) dispara intercambios de información y coordinación permanentes con servicios y programas que despliegan sus acciones de manera focalizada.

El acercamiento a les afiliades a través de les promotores contribuye a que las líneas de cuidado priorizadas por OSEP mejoren su llegada a las poblaciones objetivo, en especial a segmentos de población vulnerable o que enfrentan barreras para el acceso a los servicios. Por ejemplo, con el programa Ser Mujer se ha gestionado el traslado (en más de 60 viajes) de afiliadas que viven en localidades distantes al centro de atención de la mujer ubicado en la Ciudad de Mendoza, donde en una misma jornada realizan todos los estudios diagnósticos para la detección precoz de cáncer femenino y pueden participar en talleres de Salud Reproductiva y/o Violencia de Género, según sus intereses. Parte de las mujeres a las que convocamos accedían por primera vez en su vida a estudios como una mamografía. Estimamos que el "efecto grupo" de esta actividad, que es posible gracias a la coordinación con equipos locales de OSEP y con municipios, minimiza algunos de los temores y mitos que suelen operan como barrera subjetiva para realizar esos estudios y resulta multiplicador entre pares, una vez que las mujeres regresan a los territorios.

Por otra parte, a partir de la nominalización de los niños de 0 a 2 años que no registraban consumos en salud en el último año en el sistema de OSEP, se contacta a las familias y se las orienta hacia servicios de proximi- 
dad, como las Unidades de Atención Familiar o los prestadores preferenciales ${ }^{20}$ del programa Crecer. En tanto, las situaciones de mayor vulnerabilidad se trabajan con el equipo que trata a niñes y adolescentes con necesidades especiales de salud (NANEAS). En departamentos alejados de la Ciudad de Mendoza se ha intervenido en situaciones críticas de niñes con discapacidad y se ha remitido al Programa de Atención Temprana a chiques que, en los relevamientos, se identificó que presentaban alguna alteración en el desarrollo a partir de preguntas disparadoras muy simples como la edad en la que habían comenzado a caminar y/o a sentarse.

La relación de nuestro equipo con las Unidades de Atención Familiar OSEP Cerca y las sedes regionales, que son los dispositivos del primer nivel de atención de propiedad de la Obra Social, está condicionada por la predisposición diferencial de los grupos de trabajo a integrar a sus tareas cotidianas los aportes de les promotores de salud y a incorporar lineamientos del enfoque poblacional. Nos referimos, por ejemplo, a la búsqueda activa de asegurados con criterios de vulnerabilidad y la responsabilidad por las poblaciones de referencia, aun cuando no expresen activamente sus necesidades y demandas. Este tipo de procesos supone revisar lógicas de trabajo internas, como los modos en que circula la información clínica y administrativa, y las relaciones verticales y horizontales entre programas, servicios y áreas que deciden sobre los recursos de la obra social y sus formas de llegada a les asegurades.

En otro orden, hemos avanzado en la intersectorialidad, coordinando acciones con organizaciones que inciden en los determinantes sociales de la salud. Los ASIS permiten identificar problemas que condicionan la salud de los afiliados y que involucran decisiones de poder político provincial y municipal. A modo de ejemplo, acordamos con la Dirección Provincial de Transporte disponer de información de primera mano sobre los cambios en los recorridos de micros que conectan a afiliades con los principales centros asistenciales; establecimos caminos con el Área Mujer del Poder Judicial para

$20 \mathrm{El}$ prestador preferencial es una figura que responde a la idea de jerarquizar en la cartilla de prestadores (y ofrecer un pago diferencial) a les profesionales que entre otros requisitos completan la historia clínica electrónica, prescriben on-line y respetan las guías de práctica aprobadas por la organización 
atender temas de violencia de género y, con la Dirección de Escuelas, para favorecer la inserción de adolescentes excluidos del sistema educativo.

2. Parte de la información que producimos es contemplada en diferentes niveles de la organización para tomar decisiones sobre los modos de brindar servicios.

Responsables de diseñar convenios, de planificación, gestores territoriales, coordinadores de programas, directives y encargades de servicios de los hospitales propios y de algunos de los equipos de salud de proximidad nos demandan y/o se valen de nuestros informes para incorporar a la organización de sus tareas aspectos de la lógica territorial/poblacional.

Resultan especialmente valorados los informes sobre perfiles sociodemográficos de les afiliades y los mapeos de patrones de utilización de servicios por zonas y por segmentos de edad. A partir de demandas puntuales, hemos construido reportes que operan como insumos para identificar la consistencia entre la oferta de prestaciones convenida por OSEP en los territorios y la disponibilidad real de servicios para diferentes poblaciones. Si bien no estamos en condiciones de ofrecer representatividad estadística, porque priorizamos barrios y poblaciones vulnerables, entendemos que la información que producimos interpela la orientación de algunas políticas asistenciales y la adecuación de servicios a realidades de usuaries no del todo visibilizadas, como es la situación de las personas mayores. Por ejemplo, identificamos que cerca de un tercio de las familias contactadas tiene entre sus integrantes a una persona adulta mayor; que $8 \%$ de las personas afiliadas de este segmento de edad viven solas y que casi $5 \%$ de les mayores de 65 años (13,7\% del padrón total) no tendrían a quién recurrir si se les presentara un problema de salud imprevisto. La no utilización de servicios de salud en este grupo de edad es desigual en los territorios. Está en el orden del 17\% en el departamento (urbano) de Guaymallén y del 10\% en Santa Rosa (rural). Esta información ha reorientado determinados dispositivos de ayuda que brinda la obra social, que partían de creencias erróneas, como que les adultes mayores son hiper consumidores de atención médica o que siempre cuentan con alguien que puede hacer gestiones en su nombre. 
También hemos construido indicadores que dan cuenta de desigualdades territoriales en términos de determinantes sociales de la salud y de utilización de servicios. Sabemos, por ejemplo, que 16\% de las familias no posee vivienda propia, que 30\% reside en viviendas deficitarias y que este problema se expresa de manera diferente según las zonas: trepa a $46 \%$ en barrios populares de Las Heras y está en el orden del 7\% en los distritos alejados de la ciudad de San Rafael.

Por otro lado, los mapeos de activos sociales posibilitan detectar organizaciones, como centros de jubilados o centros culturales y espacios públicos para la recreación y la convivencia que, desde la concepción salutogénica (Hernán, Morgan y Mena, 2013), colaboran para que las familias se apropien de recursos que pueden favorecer su bienestar y para que los equipos de salud incorporen a esas organizaciones en estrategias de cuidado específicas. Con la construcción de las guías de activos, hacemos visibles recursos locales y, "al poner énfasis en las fortalezas personales, familiares y sociales que pueden contribuir a resolver necesidades concretas en salud de una manera integral superando el enfoque centrado únicamente en el riesgo" (Betancurth Loaiza, Vélez Álvarez y Sánchez Palacio, 2020:149). Además, les aportamos a les profesionales una herramienta concreta al servicio de la prescripción social21.

3. Hemos diseñado una metodología de trabajo que puede extenderse al conjunto de la organización.

Desplegar la estrategia poblacional nos demanda espacios de capacitación permanente como equipo en las nociones teóricas que orientan la tarea, en el diseño de procesos de inserción territorial y su adaptación a diferentes contextos, en metodologías para la obtención de información secundaria y primaria de calidad y en la carga y análisis de múltiples fuentes de infor-

21 El término prescripción social se deriva del inglés social prescribing y se refiere al proceso por el cual un integrante del equipo de salud recomienda un activo de la comunidad al usuario para mejorar su salud o resolver un problema. No es lo mismo que un médico de familia le recomiende a un adulto mayor sin apoyo social que "busque relacionarse con otros" de manera vaga, que le facilite en el contexto de la consulta los espacios y horarios donde se congregan sus pares para aprender danzas, hacer yoga o compartir caminatas. 
mación. Tenemos encuentros sistemáticos de capacitación con los equipos territoriales en tópicos como el género como determinante de la salud, en nociones de epidemiología, promoción de la salud como base en activos sociales, trabajo en grupos, competencias comunicacionales y normalización de informes, entre otros. Para apoyar estos procesos, hemos producido una serie de materiales, entre ellos un Glosario de Términos de Promoción Territorial de la Salud, inicialmente para quienes trabajan en la estrategia, pero que también está a disposición de todes les trabajadores de la obra social.

En el tercer año de trabajo, formalizamos la rotación en nuestra área de residentes de Medicina Familiar, de Trabajo Social en Salud Pública y de estudiantes de pregrado de Sociología. Residentes de Clínica Médica y de Salud Mental han solicitado instancias de formación para incorporar a sus currículas la capacitación en salud poblacional.

\section{Reflexiones finales}

Avanzada la experiencia, sostenemos que es posible desplegar el enfoque territorial-poblacional cuando se conjuga una decisión de política institucional con el compromiso sostenido de un equipo con diferentes formaciones disciplinares e interés en trabajar para hacer visibles, al interior de la organización, las desiguales condiciones de vida que influyen en la salud de las familias.

Entendemos que, además de aportar conocimientos, hemos desarrollado experticias de intervención que colaboran para que los servicios de salud en el primer nivel de atención sean un poco más accesibles desde el punto de vista sociocultural, es decir, que ajusten sus características y modalidades (horarios de atención, perfil de les profesionales responsables, organización de las agendas de admisiones y turnos) "a las características, necesidades, situaciones, particularidades y marcos de sentido de las poblaciones destinatarias” (Landini, González Cowes y D’Amore, 2014:232).

No menospreciamos los resultados que hemos alcanzado, pero sabemos que estamos lejos de que la organización, en su conjunto, se haga eco de que el territorio no es un accidente en la vida, la salud y la enfermedad de las poblaciones (Tobar, 2010) y pueda incorporar que afecta tanto las condicio- 
nes de vida y de salud de los afiliados como los modos concretos de diseñar y brindar servicios sanitarios. Entendemos que solo en la medida que se comprenda el territorio como espacio donde se expresan las desiguales posibilidades de las poblaciones de apropiarse de recursos para construir salud (Molina Jaramillo, 2018), se podrán reorientar las prioridades de financiamiento, de gestión y la organización asistencial atendiendo a esas diferencias.

En la tarea cotidiana, constatamos que considerar a las familias y a los microterritorios (y no a los individuos) como nuestras unidades de intervención desencadena, necesariamente, el trabajo intersectorial (Tobar, 2008). En este punto, y dada la gravitación de OSEP en el contexto provincial, reconocemos avances prometedores. Tanto en la línea que sugieren la estrategia $S a$ lud en todas las politicas (Ståhl, Wismar, Ollila, Lahtinen \& Leppo, 2006) como en la incipiente adopción de la perspectiva salutogénica, que enfatiza el valor de las capacidades locales en la construcción de la salud (García et al, 2019).

Nos consta que, al operar como nexos entre las necesidades de salud de las familias, los servicios (en especial los del primer nivel de atención) y los recursos locales con incidencia en los determinantes sociales de salud, les promotores territoriales son agentes clave para avanzar en el enfoque territorial y poblacional. Pero no pueden trabajar a modo de islas. Como señalan Cabral y Abramovici (2018) en una reflexión sobre el papel de promotoras de salud en el partido de Moreno, Buenos Aires, advertimos que les profesionales no siempre son receptives a saberes y metodologías que desbordan la biomedicina. A los equipos de los servicios, programas y áreas administrativas de la organización les cuesta correrse de lógicas instrumentales donde el eje es el individuo (o una parte de su cuerpo) descontextualizado, y aceptar que la mediación de la o el promotor territorial, en contacto directo con los modos como las familias perciben y tramitan la salud de sus integrantes, puede interrogar sus prácticas y sus rutinas. Puertas adentro, entonces, debemos seguir revisando las lógicas gestionarias y asistenciales centradas en la oferta y en el combate de enfermedades puntuales que dificultan la construcción de las redes de trabajo, que son las que posibilitan la coordinación asistencial (Fernández Moyano y Ollero Baturone, 2010). 
Asumimos, con Tobar (2010), que la responsabilidad nominada de los equipos por los resultados de salud de poblaciones que habitan territorios concretos ofrece potencialidades "para cambiar integralmente el modelo de atención”. Por lo que avanzar en identificarlas correctamente, saber cómo se distribuyen, de qué enferman o pueden enfermar esas poblaciones y los modos en que utilizan o no los servicios resultan insumos innegables para imprimir racionalidad y equidad en la seguridad social. Hemos comprobado, no obstante, que investigar y transferir resultados en esta línea no desencadena automáticamente procesos para poner en sintonía oferta con necesidades (Gómez, Orozco, Rodríguez y Velázquez, 2006). Por un lado, porque apostar por una estrategia territorial poblacional en el contexto de un sistema de provisión altamente fragmentado, con cuidados discontinuos, poco equitativo y poco eficiente (Giovanella et al, 2015) supone una fuerte revisión de los principios desde los que operan todes les actores de ese sistema, incluso les asegurades. Por el otro lado, porque la decisión de regular la oferta en base a necesidades, en vez de habilitar servicios de manera indiscriminada, que es una condición básica de los modelos de gestión territorial, de acuerdo con Tobar (2010), implica reorientar políticas y recursos. Exige nuevos aprendizajes puertas adentro de la organización y lidiar con los intereses de las corporaciones que gravitan en la seguridad social.

Sin perder de vista los desafíos y las tensiones que supone este camino, optamos por compartir de manera sostenida los conocimientos que vamos adquiriendo, así como nuestros errores y aciertos en los procesos mediante los que nos vinculamos con las poblaciones en los espacios en los que viven y/o trabajan, porque entendemos que este es el modo de imprimirle viabilidad y sostenibilidad a la estrategia en la organización de salud en la que trabajamos.

\section{Referencias bibliográficas}

Araujo González, Rafael. (2015). Vulnerabilidad y riesgo en salud: ¿dos conceptos concomitantes? Revista Novedades en Población, 11(21), 89-96.

Betancurth Loaiza, Diana; Vélez Álvarez, Consuelo, y Sánchez Palacio, Natalia (2020). Cartografía social: construyendo territorio a partir de los activos comunitarios en salud. Entramado, 16 (1), 138-151. 
Borde, Elis. y Torres-Tovar, Mauricio (2017). El territorio como categoría fundamental para el campo de la salud pública. Saúde Debate, 41 (2), 264-275.

Cabral Natalia. y Abramovici, David. (2018). Salud y promoción territorial: reflexiones a partir de una experiencia con promotoras de salud. Revista de Políticas Sociales 5(6), 131-136. http://www.rps.unm.edu.ar/ojs/index.php/rps/article/view/48

Cetrángolo, Oscar y Goldschmit, Ariela (2018). Las obras sociales y otras instituciones de la Seguridad social para la salud en Argentina. Origen y situación actual de un sistema altamente desigual. Ciudad de Buenos Aires, Argentina, Fundación CECE. Recuperado de: http://fcece.org.ar/wp-content/uploads/informes/obras-sociales-argentina.pdf

Chiara Magdalena (2016). Territorio, políticas públicas y salud. Hacia la construcción de un enfoque multidimensional para la investigación. Revista Gerencia y Políticas de Salud, 15(30), $10-22$.

De Ponti, Hector, Canale, Sandra y Monteferrario, Mariano.(2016). Fragmentación de cobertura de servicios de salud. El caso de las Obras Sociales Provinciales. Jornadas de Economía de la salud 2016/ XXV Jornadas internacionales / XXVI Jornadas nacionales "Crisis económica, inversión y sustentabilidad del sector salud en Latinoamérica y Caribe". Universidad ISALUD. Ciudad Autónoma de Buenos Aires, Argentina. Recuperado de: http://www.oossp.com.ar/archivos/pubs/ fragmentac $\% 20 \mathrm{cob}^{2} \% 20 \mathrm{y} \% 20 \mathrm{osp} \% 20 \mathrm{aes} \% 202016$.pdf

Fernández Moyano, Antonio y Ollero Baturone, Manuel (2010). Percepción de la continuidad asistencial: Conocer para actuar. Rev. Española de Salud Pública, 84(4), 349-351.

García, Mariano; Miota, Adrian; Barranco Tirado, Juan; Belda Grindley, Carlos; Páez Muñoz, Eva; Rodríguez Gómez, Susana y Lafuente Robles, Nieves (2019). Salud Comunitaria Basada en activos. Guía breve. Granada, Escuela Andaluza de Salud Pública y Servicio Andaluz de Salud. Recuperado de: https://www.easp.es/wp-content/uploads/dlm_uploads/2019/01/ EASP Guia breve SaludComActivos-FUM-05-12-18.pdf

Giovanella, Ligia; Almeida, Patyy; Romero, Roman; Oliveira, Suelen y Silva, Herland (2015). Panorama de la Atención Primaria de Salud en América Latina: concepciones, componentes y desafíos. Saúde debate, 39(105), 300-322.

Gómez, Ruben; Orozco, Deisy; Rodríguez, Fabio y Velázquez, Willian (2006). Políticas públicas y salud: relación entre investigación y decisión. Revista Fac. Nac. Salud Pública, 24(2), 105-118.

Landini, Fernando; González Cowes, Valeria. y D’Amore, Eliana. (2014). Hacia un marco conceptual para repensar la accesibilidad cultural. Cad. Saúde Pública, 30 (2), 231-244.

López Arellano, Oivia; Escudero, Jose y Dary Carmona, Luz (2008). Los determinantes sociales de la salud. Una perspectiva desde el Taller Latinoamericano de Determinantes Sociales de la Salud, ALAMES. Medicina Social, 3 (4), 28-36. http://www.medicinasocial.info/index. $\mathrm{php} / \mathrm{medicinasocial} /$ article/view/260

Menéndez, Eduardo (1990). El modelo médico hegemónico. Estructura, función y crisis. En E. Menéndez (Ed.) Morir de alcohol. Saber y hegemonía médica. México, Alianza Editorial Mexicana.

Ministerio de Salud de la Nación (2018). Análisis de situación de salud República Argentina. Ciudad Autónoma de Buenos Aires, Argentina, Ministerio de Salud de la Nación. 
Molina Jaramillo, Astrid (2018). Territorio, lugares y salud: redimensionar lo espacial en salud pública Cad. Saúde Pública, 34(1). https://doi.org/10.1590/0102-311x00075117

Hernán, Mariano; Morgan, Antony y Mena, Angel (2013). Formación en salutogénesis y activos para la salud. Monografías EASP, (51).

Pría Barros, María (2006). "Análisis de la situación de salud en áreas pequeñas". En S. Martínez Calvo, M. Caraballoso Hernández, M. Astraín Rodríguez, M. Pría Barros, I. Perdomo Victoria y C. Arocha Mariño (Ed.), Análisis de la situación de salud. (pp. 105-122). La Habana, Cuba, Editorial Ciencias Médicas.

Ståhl, Timo; Wismar, Mathhias; Ollila, Eeva; Lahtinen Eero y Leppo, Kimmo (2006). Health in All Policies. Prospects and potentials. Finland, Ministry of Social Affairs and Health and European Observatory on Health Systems and Policies.

Tobar Federico (2010). "Gestión territorial en salud”. En M. Chiara y J. Moro (Eds.), Pensando la agenda de la política. Capacidades, problemas y desafíos(pp.15- 44). Buenos Aires, Argentina, Instituto del Conurbano, Universidad Nacional General Sarmiento.

Tobar, Federico (2008). Cambios de paradigma en Salud Pública. XII Congreso del CLAD. Buenos Aires, Argentina. Recuperado de: http://www.fsg.org.ar/maestria pdfs/3.pdf

Recibido: 27/03/2020

Aceptado: $18 / 11 / 2020$ 